\title{
Rôle de la nutrition minérale et de l'approvisionnement en eau sur la croissance du pin laricio de Corse dans l'ouest de la France
}

\author{
F Lebourgeois $^{1 *}$, G Lévy $^{2}$, M Becker $^{1}$, Y Lefevre $^{2}$ \\ ${ }^{l}$ Équipe phytoécologie ; 'Équipe sol et nutrition, unité d'écophysiologie forestière, \\ Inra-Nancy, 54280 Champenoux, France
}

(Reçu le 6 décembre 1995 ; accepté le 13 février 1996)

\begin{abstract}
Summary - Effects of mineral nutrition and water site conditions on radial growth of Corsican pine in western France. The importance of mineral nutrition and soil water regime (water soil reserve index and lateral water supply) for the radial growth of 1808 mature Corsican pines (Pinus nigra Arnold ssp laricio Poiret var corsicana) (average age $=30$ years) sampled in 183 sites in western France (Pays de la Loire region) was studied. Foliage and chemical soil analyses indicate that growth depends exclusively on phosphorus nutrition. The optimal $\mathrm{P}$ foliar concentration is $0.15 \%$ (N/P foliage ratio $=10)$, the critical value is $0.12 \%(\mathrm{~N} / \mathrm{P}=14)$ and the deficiency level is $0.10 \%(\mathrm{~N} / \mathrm{P}=18)$. Needle content of other nutrients is sufficient for tree requirements and does not differ between trees with different growth levels. The average concentrations are $1.59 \%, 0.19 \%, 0.12 \%$ and $0.59 \%$ for $\mathrm{N}$, $\mathrm{Ca}, \mathrm{Mg}, \mathrm{K}$, respectively. The response of trees to phosphorus is greater on moist sites than on dry sites, but considerably decreases during drought periods. Soil water regime, which is found to predispose trees to climatic variations, may locally accentuate the effects of exceptionally dry years and hence induce durable radial growth decrease and subsequently forest decline. However, low competition between trees seems to be able to decrease the intensity of dryness by minimizing water competition within stands. In order to improve the radial growth of Corsican pine plantations, the best advice is to avoid this species on sites clearly unfavourable for mineral nutrition and/or for water supply, and to apply an adequate silviculture (regular thinnings) in order to minimize water competition between trees, especially during climate crisis.
\end{abstract}

Corsican pine / dendrochronology / radial growth / nutrition / phosphorus / water supply / drought

Résumé - La caractérisation physicochimique des sols, l'analyse de la nutrition foliaire et des conditions locales d'approvisionnement en eau (indice de réserve en eau du sol et classe de drainage) ont permis de mettre en évidence le rôle essentiel du phosphore et de l'alimentation en eau sur la crois-

* Correspondance et tirés à part

Tél. : (33) 0383394041 ; fax : (33) 0383394022 ; courriel : lebourg@nancy.inra.fr 
sance radiale de 1808 pins laricio de Corse adultes (âge moyen $=30$ ans) (Pinus nigra Arnold ssp laricio Poiret var corsicana) échantillonnés dans 183 placettes de l'Ouest de la France (région Paysde-la-Loire). La teneur foliaire en phosphore associée au niveau de croissance le plus élevé correspond à une valeur de $0,15 \%$ (rapport $\mathrm{N} / \mathrm{P}=10$ ), la croissance moyenne à une teneur de $0,12 \%$ (rapport $\mathrm{N} / \mathrm{P}=14$ ) et la croissance la plus faible à une valeur de $0,10 \%($ rapport $\mathrm{N} / \mathrm{P}=18)$. Pour les autres éléments, les valeurs observées sont suffisantes pour les besoins des peuplements (teneurs optimales à suboptimales). Les valeurs moyennes sont respectivement de $1,59 \%$ pour l' $\mathrm{N}, 0,19 \%$ pour le Ca, $0,12 \%$ pour le $\mathrm{Mg}$ et $0,59 \%$ pour le K. L'effet sur la croissance de la nutrition phosphorée est d'autant plus important que l'alimentation locale en eau est bonne, mais est fortement inhibé lors de périodes à déficits pluviométriques marqués. La disponibilité en eau des sols, qui prédispose les peuplements aux effets des variations climatiques, et plus particulièrement pluviométriques, peut localement accentuer les effets des sécheresses et entraîner des réductions importantes de croissance. Pour les peuplements correspondants, il est possible que cela se traduise, à plus ou moins long terme, par un véritable dépérissement. Cependant, une moindre concurrence entre arbres au sein des peuplements semble pouvoir compenser en partie les effets d'une alimentation hydrique limitante, et permettre de minimiser la compétition pour l'eau dans le cas de sécheresses durables. En ce qui concerne les recommandations aux gestionnaires actuels et futurs des peuplements forestiers, il serait souhaitable de limiter les reboisements de pin laricio de Corse aux stations dont l'alimentation minérale et/ou hydrique n'est pas un facteur limitant essentiel, et adopter un régime sylvicole adéquat afin de maintenir un état de concurrence raisonnable et, si possible, adapté aux conditions d'approvisionnement en eau.

\section{pin laricio / dendrochronologie / croissance radiale / nutrition / phosphore / alimentation en eau / sécheresse}

\section{INTRODUCTION}

Introduit en France continentale à la fin du XVIII ${ }^{\mathrm{e}}$ siècle, c'est seulement à partir des années 1950 que le pin laricio de Corse (Pinus nigra Arnold ssp laricio Poiret var corsicana) a connu son véritable essor avec plus de 40000 ha plantés en 30 ans (RomanAmat, 1984). À l'heure actuelle, cette espèce, très prisée pour son niveau de production et la qualité de son bois, occupe la troisième place des espèces résineuses de boisement et reboisement au niveau national, et la première dans l'Ouest et le Centre de la France (Durel et Charon, 1994). Les aptitudes connues du pin laricio dans son aire naturelle (Debazac, 1964), liées à ses origines bioclimatiques (montagnes méditerranéennes), permettent de penser que ce choix peut être judicieux dans ces régions. Il est par ailleurs probable que son utilisation prenne une importance grandissante dans le contexte actuel de la déprise de nombreuses terres agricoles.
Paradoxalement, notre connaissance du comportement de ce résineux sur le continent, et particulièrement dans le contexte pédoclimatique de l'Ouest de la France est encore imprécise, et en tout cas insuffisante pour répondre de façon sûre aux interrogations des gestionnaires des peuplements actuels, et à celles des reboiseurs potentiels. Généralement, on recommande le pin laricio sur les sols siliceux sains en évitant les stations très hydromorphes ou très carbonatées (Roman-Amat et Arbez, 1985). Au niveau minéral, ses exigences semblent réduites. Sur des sols lessivés à pseudogley argileux, pauvres et mal drainés (forêt de Moulière, Vienne), les travaux de Bonneau (1963, 1970), Ranger (1978, 1981) ont montré qu'un apport d'acide phosphorique, associé au calcium et potassium, permet un doublement de la production primaire de l'origine à 15 ans. En Sologne, sur un sol sableux pauvre du type brun acide peu évolué et en région méditerranéenne sur sols calcaires, les travaux de Nys et al (1985) et 
Guitton et al (1988) sur des jeunes peuplements ont montré qu'une fertilisation associant l'azote et le phosphore donne un gain d'accroissement significatif. En Écosse, sur des dunes sableuses, Proe et al (1992) ont montré qu'une fertilisation azotée sur des peuplements adultes permet, selon la dose appliquée, une augmentation de la surface terrière de 35 à $107 \%, 10$ à 15 ans après le traitement. Sur des sols podzoliques à gley, les travaux de Moffat et al (1991) ont montré que l'apport de phosphore par l'intermédiaire d'un épandage de boues d'égout $\left(200 \mathrm{~m}^{3} / \mathrm{ha}\right)$ permet une augmentation du volume de bois de $50 \%$ sur des arbres de 35 ans.

Afin de préciser le comportement de ce résineux dans le secteur ligérien, et de fournir à terme aux organismes chargés du développement les éléments leur permettant de guider efficacement les propriétaires forestiers dans le choix des stations à reboiser, une étude autécologique a été menée dans la région Pays-de-la-Loire (Lebourgeois, 1995).

Pour étudier le rôle des paramètres stationnels sur la croissance, la démarche la plus classique consiste à raisonner en termes de production, c'est-à-dire définir pour chaque site échantillonné un indice de fertilité (hauteur dominante du peuplement correspondant à l'âge de référence fixé) qui est supposé varier entre stations dans le même sens que la production (Décourt, 1973). Si cet indice permet de mettre en évidence et de hiérarchiser les principaux facteurs explicatifs des différences de production observées, il ne permet pas une approche dynamique, c'est-à-dire temporelle, des contraintes de croissance subies par les arbres. Cet indice ne permet pas de dater précisément d'éventuels changements de comportement des peuplements au cours du temps, et ne tient pas compte du climat dont le signal est enregistré annuellement par l'arbre (Fritts, 1976 ; Tessier, 1989), et qui peut modifier la réaction des peuplements à certains paramètres du milieu, comme par exemple la nutrition minérale (Smith et al, 1977; Turner, 1982 ; Raison et al, 1990 ; Yarie et al, 1990).

Ainsi, afin de combiner les dimensions spatiales et temporelles des phénomènes étudiés, c'est l'approche dendroécologique, associant la mesure de la croissance radiale et l'analyse des conditions physiques et biotiques de croissance, qui a été privilégiée dans cette étude (Lebourgeois, 1995).

Cet article présente les résultats relatifs au rôle de la nutrition minérale et des conditions d'approvisionnement en eau sur la croissance radiale de peuplements adultes de pin laricio de Corse dans l'Ouest de la France.

\section{MATÉRIEL ET MÉTHODES}

\section{Aire d'étude et échantillonnage}

Un total de 1808 pins a été échantillonné dans 183 peuplements purs de pin laricio de Corse. Les placettes ont été installées dans trois des cinq départements de la région Pays-de-la-Loire de façon à couvrir la gamme stationnelle la plus large possible. Sur chaque site, dix arbres dominants bien conformés ont été carottés à la moelle à $1,30 \mathrm{~m}$ du sol à l'aide d'une tarière de Pressler de $5 \mathrm{~mm}$ de diamètre intérieur, puis mesurés (hauteur totale $(\mathrm{H})$ et diamètre $(\mathrm{D})$ à $1,30 \mathrm{~m}$ ). Le facteur d'élancement $f$ a été ensuite calculé comme le rapport de la hauteur $\mathrm{H}$ sur le diamètre $\mathrm{D}(f=\mathrm{H} / \mathrm{D})$. Ce facteur n'est pas uniquement un coefficient de forme, mais renseigne également sur la position sociale des arbres dans le peuplement (Zarnovican et Trencia, 1987 ; Ung, 1989). On considère généralement qu'il est un bon intégrateur des conditions moyennes de concurrence subies par l'arbre tout au long de sa vie. Les arbres dominants ou codominants ont un facteur d'élancement inférieur à 100 , et pour un peuplement, plus le facteur d'élancement est faible $(<80)$, plus le peuplement est stable (Pardé et Bouchon, 1988). Le rapport H/D, s'il permet de différencier les classes sociales d'un peuplement, n'est cependant pas indépendant de l'âge. Ainsi, c'est seulement pour des peuplements d'âges peu différents que la comparaison des valeurs des 


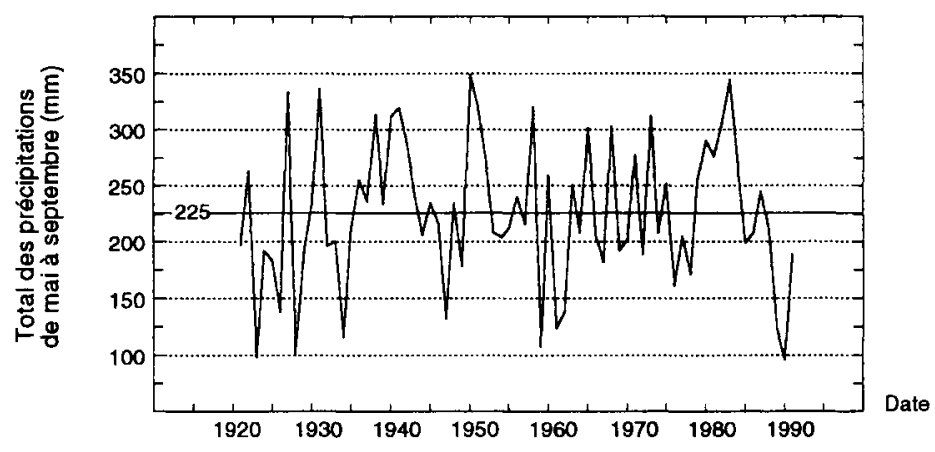

Fig 1. Totaux pluviométriques (en $\mathrm{mm}$ ) de mai à septembre (saison de végétation) au poste météorologique d'Angers. Le trait plein présente la normale (en mm) calculée sur la période 1921-1991.

facteurs d'élancement entre arbres peut se faire directement. Dans le cas contraire, il est nécessaire d'étudier préalablement la relation liant le facteur d'élancement à l'âge (Lebourgeois, 1995).

Les plus anciennes plantations qui ont pu être échantillonnées ont au plus 70 ans (à $1,30 \mathrm{~m}$ ), et l'âge moyen des 1808 pins étudiés est de 30 ans (Lebourgeois et Becker, 1996).

La région Pays-de-la-Loire est une zone de transition entre climat océanique et semi-continental. À Angers, station la plus représentative du climat régional et pour laquelle on dispose d'une longue série climatique continue et homogène (1921-1991), la température moyenne annuelle est de $11,5^{\circ} \mathrm{C}$ avec un maximum en juillet $\left(19^{\circ} \mathrm{C}\right.$ ) et un minimum en janvier $\left(4,6^{\circ} \mathrm{C}\right)$. Les précipitations totales sont assez faibles $(601 \mathrm{~mm})$, mais bien réparties sur l'ensemble de l'année (40 à $60 \mathrm{~mm}$ par mois). Pour la période de mai à septembre (saison de végétation), la moyenne est de $225 \mathrm{~mm}$ (fig 1). Pour cette période, les faits récents les plus remarquables sont les déficits importants du début des années 1960 (respectivement $-118,-102$ et $-82 \mathrm{~mm}$ par rapport à la normale pour 1959, 1961 et 1962), la sécheresse de $1976(-64 \mathrm{~mm})$, et la diminution très importante et presque continue depuis 1983 des précipitations printanières et estivales, particulièrement marquée en 1989 et 1990. L'année 1990 est d'ailleurs l'année « record» avec un déficit de $129 \mathrm{~mm}$ pour la saison de végétation, et de $141 \mathrm{~mm}$ sur l'ensemble de l'année par rapport aux normales. L'année 1969 est également une année particulière, avec une sécheresse très marquée en juin, juillet et août (précipitation totale de $53 \mathrm{~mm}$ pour une normale de $125 \mathrm{~mm}$ ).

\section{Les descripteurs stationnels}

Chaque placette a été caractérisée par les descripteurs classiques : pente (en \%), exposition, position topographique. La classe de drainage a également été notée car elle permet d'estimer les conditions d'apports latéraux d'eau par ruissellement au niveau de la placette (Le Goff et Lévy, 1984). Dans la réalité, la position topographique et la classe de drainage sont des paramètres étroitement liés. Une classe de drainage défavorable correspond à des situations topographiques du type « haut de pente » ou "rebord de plateau». A l'opposé, le drainage favorable est associé à une topographie du type « bas de pente » ou de "fond de vallon» (Lebourgeois, 1995).

Afin de caractériser le contexte chimique de croissance des arbres, des analyses minérales ont été réalisées sur l'horizon $A_{1}$ et sur le premier horizon minéral (entre -20 et $-30 \mathrm{~cm}$ ) de chaque sol. L'horizon $\mathrm{A}_{1}$ a été prélevé à cinq endroits différents de la placette, et le premier horizon minéral sur les parois de la fosse pédologique ouverte au centre de la placette. Pour ce dernier prélèvement, l'analyse granulométrique a également été effectuée. Les analyses ont été effectuées sur un échantillon unique regroupant les différents prélèvements, et sur la fraction inférieure à $2 \mathrm{~mm}$, obtenue après tamisage de la terre sèche. Les cations échangeables, et donc le taux de saturation $\mathrm{S} / \mathrm{T}$, ont été obtenus après échange au chlorure de cobaltihexamine $0,05 \mathrm{M}$ (c'està-dire au $\mathrm{pH}$ du sol). Les éléments analysés et les méthodes employées sont récapitulés dans le tableau I. 
Tableau I. Analyses minérales des sols et méthodes employées.

\begin{tabular}{lccc}
\hline Analyses & Méthodes & $\begin{array}{c}\text { Horizon } \\
\text { Al }\end{array}$ & $\begin{array}{c}\text { Horizon } \\
\text { minéral }\end{array}$ \\
\hline $\begin{array}{l}\text { Granulométrie cinq fractions } \\
(\mathrm{Sf}, \mathrm{Sg}, \mathrm{Lf}, \mathrm{Lg}, \mathrm{A})\end{array}$ & & $\mathrm{X}$ \\
Carbone $(\mathrm{C})$ & Anne 1945 & $\mathrm{X}$ & \\
Azote total $(\mathrm{N})$ & Kjeldahl & $\mathrm{X}$ & \\
Matière organique & & $\mathrm{X}$ & $\mathrm{X}$ \\
pH (eau) & & $\mathrm{X}$ & $\mathrm{X}$ \\
Calcium $(\mathrm{Ca})$ & cobaltihexamine & $\mathrm{X}$ & $\mathrm{X}$ \\
Magnésium $(\mathrm{Mg})$ & cobaltihexamine & $\mathrm{X}$ & $\mathrm{X}$ \\
Potassium $(\mathrm{K})$ & cobaltihexamine & $\mathrm{X}$ & $\mathrm{X}$ \\
CEC & & $\mathrm{X}$ & $\mathrm{X}$ \\
Phosphore $\left(\mathrm{P}_{2} \mathrm{O}_{5}\right)$ & Duchaufour & & \\
\hline
\end{tabular}

Les analyses ont toutes été faites par le laboratoire d'analyses des sols de l'INRA à Arras. Sf : Sable fin ; Sg : Sable grossier ; Lf : Limon fin ; Lg : Limon grossier ; A : Argile. Carbone, azote, phosphore et matière organique sont exprimés en $\mathrm{g} \cdot \mathrm{kg}^{-1}$; les cations échangeables et la CEC (capacité d'échange cationique) sont exprimées en cmol $+\mathrm{kg}^{-1}$ de terre fine.

Les analyses minérales des sols ont été complétées par des analyses foliaires. À partir des mesures de la concentration en éléments minéraux dans les aiguilles, ces analyses permettent d'apprécier la qualité de la nutrition, et de mettre en évidence d'éventuels problèmes de carence minérale. Pour des raisons financières, seules 120 placettes dans la gamme d'âge 20-30 ans ont été échantillonnées. Le choix a été guidé par le fait que c'est dans cette gamme d'âge que le nombre de placettes installées a été le plus grand, et la diversité stationnelle rencontrée la plus importante. Sur chaque site, les aiguilles de l'année de cinq des dix arbres sélectionnés ont été prélevées dans le tiers supérieur du houppier, soit un total de 600 arbres. Les prélèvements ont été groupés en un échantillon unique, et les analyses ont porté sur l'azote $(\mathrm{N})$, le calcium $(\mathrm{Ca})$, le magnésium $(\mathrm{Mg})$, le potassium $(\mathrm{K})$ et le phosphore (P). Le rapport N/P a également été calculé car il est considéré comme un excellent indicateur de l'équilibre nutritionnel des arbres (Bonneau, 1988 ; Mesanza et al, 1993).

Un indice de réserve utile en eau (Iru) a été calculé pour chaque placette. En raison de la diversité des situations rencontrées, il a fallu fixer des critères pour son estimation, et notamment la profondeur de sol sur laquelle allait être calculé cet indice de disponibilité en eau. À partir de la description du profil et de l'observation du système racinaire au niveau de chaque horizon décrit (taille et densité de racines), les éléments suivants sont apparus être particulièrement limitants pour le développement racinaire : une teneur en cailloux supérieure à $50 \%$; une hydromorphie très prononcée ( $\geq 50 \%$ de taches rouilles); une texture argileuse lourde ( $\geq 45 \%$ d'argile); un horizon très compact ou fortement tassé (alios, roche-mère...). On peut penser que si ces éléments n'arrêtent pas totalement le passage des racines, leur densité en profondeur sera insuffisante pour que le prélèvement de l'eau à leur niveau influence significativement la croissance des arbres. Dans le cas d'absence d'un de ces facteurs limitants, l'indice de réserve utile a été calculé pour une profondeur de sol de $150 \mathrm{~cm}$ (profondeur maximale observée de l'enracinement).

Pour chaque horizon décrit, un indice «partiel » est calculé en tenant compte de son épaisseur, de sa texture (à laquelle est associée un coefficient spécifique) et de la quantité d'éléments grossiers (Lebourgeois, 1995). Pour notre étude, nous avons utilisé les coefficients de texture résultant de la synthèse bibliographique de Duhamel (1984) (tableau II). L'indice de réserve utile du sol (Iru) est alors la somme des indices partiels calculés pour chaque horizon. 
Tableau II. Coefficients de texture utilisés pour le calcul de l'indice de réserve utile en eau.

\begin{tabular}{lc}
\hline Texture & Coefficient \\
\hline Sg & 0,38 \\
Sf ou SgL & 0,58 \\
LS & 0,77 \\
Ls & 0,85 \\
L & 1,00 \\
LSA & 1,00 \\
SA & 1,00 \\
Al & 0,90 \\
As & 0,85 \\
A & 0,80 \\
La & 1,15 \\
LA & 1,31
\end{tabular}

Sg : sable grossier ; Sf : sable fin ; L : limon ; A : argile. Excepté pour $\mathrm{Sg}$ et $\mathrm{Sf}$, la taille des lettres indique la prépondérance d'un élément sur l'autre. Ces coefficients sont des valeurs relatives par rapport à l'indice 1 des limons (Duhamel, 1984).

Pour les sols caractérisés par les successions texturales limon sur argile ou sable sur argile, l'indice de réserve utile a été corrigé en ajoutant 50 afin de tenir compte de la forte densité racinaire que l'on a pu observer au-dessus des argiles ; ces racines bénéficient d'une partie de l'eau contenue dans les argiles par remontées capillaires.

En résumé, l'indice calculé par cette méthode ne permet pas de quantifier réellement l'eau disponible dans le sol, mais permet, grâce à l'application de la même méthode de calcul pour tous les sites, de comparer et de classer les différentes stations les unes par rapport aux autres quant à la disponibilité en eau pour les arbres.

\section{Mesure de la croissance radiale}

Avant la mesure des accroissements radiaux, les 1808 carottes ont été « planées " à l'aide d'une lame tranchante, afin d'assurer une parfaite observation des moindres détails de la structure du bois, et de mieux révéler d'éventuels cernes très fins. Puis, les largeurs des cernes $(n=49379)$ ont été mesurées avec précision $(1 / 100 \mathrm{~mm})$ grâce à un système vidéo-informatisé spécifique, puis converties en accroissement en surface terrière (surface en $\mathrm{cm}^{2}$ de l'anneau correspondant à chaque cerne). Cette conversion permet de minimiser l'effet du vieillissement biologique normal de l'arbre sur l'élaboration des cernes, et elle est plus directement proportionnelle à la croissance en volume et à la quantité de bois produite annuellement par l'arbre (Briffa, 1992; Visser, 1995). Après vérification de la datation parfaite de chaque cerne (= interdatation), chaque valeur d'accroissement annuel est standardisée, c'est-à-dire convertie en indice de croissance (Ic exprimé en pourcentage). Cette standardisation se fait par référence à l'ajustement, en fonction de l'âge courant (= âge de l'arbre au moment où le cerne a été élaboré), de la courbe moyenne de tous les accroissement annuels disponibles (Lebourgeois et Becker, 1996). Cette étape permet d'éliminer la part de variation des surfaces de cernes qui demeure liée à l'âge courant, et ainsi de comparer des cernes élaborés à la même date mais d'âges courants différents (Fritts, 1976 ; Becker, 1989 ; Dupouey et al, 1992 ; Badeau et al, 1995).

Diverses courbes peuvent être alors obtenues en faisant des moyennes d'indices de croissance par date : une courbe de croissance radiale moyenne (courbe de référence) qui est la résultante de l'ensemble des facteurs écologiques qui conditionnent la croissance de l'ensemble des arbres de l'échantillon (Lebourgeois et Becker, 1996) ; un ensemble de courbes moyennes partielles, obtenues en stratifiant l'échantillon global selon les valeurs prises par divers paramètres mesurés sur le terrain (indice de réserve utile, teneurs édaphiques et foliaires en éléments minéraux...). Dans un premier temps, les différentes strates sont choisies en se fondant sur des seuils, ou des valeurs, des facteurs étudiés que l'on suppose suffisamment différents pour entraîner des différences significatives de croissance radiale. Puis, en fonction des premiers résultats obtenus, d'autres seuils sont fixés afin d'obtenir la meilleure discrimination possible des peuplements, mais tout en conservant un effectif suffisant dans chaque strate afin que les courbes de croissance calculées aient une valeur statistique. Pour chaque strate ainsi définie, on peut également calculer un indice de croissance radiale moyen ( $\mathrm{Ic}_{m}$ exprimé en \%) qui est la moyenne des indices de croissance sur l'ensemble des dates disponibles, et qui reflète le niveau moyen d'accroissement.

Le programme utilisé permet également de calculer et de comparer statistiquement les 
valeurs moyennes des autres données stationnelles ou dendrométriques disponibles pour chacune des strates, ce qui permet d'analyser plus finement le déterminisme des variations de croissance, et de mettre en évidence d'éventuelles liaisons entre le paramètre utilisé pour la stratification et d'autres facteurs stationnels.

\section{RÉSULTATS}

\section{Analyse de la fertilité des sols}

Les valeurs moyennes des différents éléments analysés par horizon sont présentées dans le tableau III. Par rapport aux seuils de fertilité donnés par Bonneau (1980 dans Lévy, 1988), les valeurs moyennes en K dans les deux horizons analysés, et celles en $\mathrm{P}_{2} \mathrm{O}_{5}$ dans l'horizon minéral correspondent à des disponibilités réduites. En revanche, les teneurs en $\mathrm{Ca}$ et $\mathrm{Mg}$ sont optimales dans les deux horizons.

\section{Analyse de la nutrition minérale des peuplements}

La comparaison des teneurs mesurées sur nos peuplements aux normes données dans la synthèse de Bonneau (1988), permet de dire que, globalement, la nutrition minérale des arbres est satisfaisante étant donné que les valeurs moyennes observées égalent ou dépassent les teneurs optimales (tableau IV). Cependant, pour le phosphore, ce résultat moyen doit être considéré avec précaution car la teneur moyenne correspond à la limite inférieure de la fourchette optimale, et la valeur minimale à un niveau critique. Dans un tel contexte, les rapports N/P élevés (47 valeurs supérieures à 14 ; $\max =22$ ) traduisent donc principalement un déséquilibre nutritionnel lié à la qualité moyenne de la nutrition phosphorée (tableau V).

La nutrition en phosphore dépend en grande partie de la disponibilité en cet élément au niveau du sol (tableau V). Pour les cations, c'est le taux de saturation (S/T) des horizons qui apparaît le plus étroitement lié à la qualité de la nutrition pour ces éléments. En revanche, pour l'azote, aucune corrélation significative n'a pu être mise en évidence entre les caractéristiques chimiques des sols et les teneurs foliaires.

Ainsi, la faible disponibilité en phosphore au niveau du sol semble donc se traduire par une nutrition effectivement déficiente

Tableau III. Valeurs moyennes ( \pm écart type) des différents éléments analysés par horizon $\left(n_{p}=183\right.$ placettes).

\begin{tabular}{|c|c|c|c|c|}
\hline & \multicolumn{2}{|c|}{ Horizon Al } & \multicolumn{2}{|c|}{ Horizon minéral } \\
\hline $\mathrm{N}$ & \multicolumn{2}{|c|}{$2,9( \pm 1,7)$} & & \\
\hline Mat Org & \multicolumn{2}{|c|}{$99,2( \pm 63,5)$} & & \\
\hline $\mathrm{C} / \mathrm{N}$ & \multicolumn{2}{|c|}{$20,4( \pm 5,2)$} & & \\
\hline $\mathrm{pH}(\mathrm{eau})$ & \multicolumn{2}{|c|}{$4,3( \pm 0,7)$} & \multicolumn{2}{|c|}{$4,7( \pm 0,8)$} \\
\hline $\mathrm{P}_{2} \mathrm{O}_{5}$ & & & $0,05( \pm 0,06)$ & 0,06 \\
\hline $\mathrm{Ca}$ & $3,00( \pm 5,5)$ & 1,0 & $1,21( \pm 3,6)$ & $\mathbf{0 , 3 0}$ \\
\hline $\mathrm{Mg}$ & $0,81( \pm 1,6)$ & 0,6 & $0,45( \pm 1,44)$ & 0,06 \\
\hline $\mathrm{K}$ & $0,23( \pm 0,2)$ & 0,6 & $0,08( \pm 0,08)$ & 0,12 \\
\hline $\mathrm{CEC}$ & \multicolumn{2}{|c|}{$7,40( \pm 6,8)$} & \multicolumn{2}{|c|}{$3,84( \pm 4,9)$} \\
\hline $\mathrm{S} / \mathrm{T}$ & \multicolumn{2}{|c|}{$42,7( \pm 25,3)$} & \multicolumn{2}{|c|}{$25,8( \pm 26,8)$} \\
\hline
\end{tabular}

Les teneurs en $\mathrm{N}, \mathrm{P}_{2} \mathrm{O}_{5}$ et matière organique sont exprimées en $\mathrm{g} \cdot \mathrm{kg}^{-1}$, et celles en $\mathrm{Ca}, \mathrm{Mg}, \mathrm{K}$ et $\mathrm{CEC} \mathrm{en} \mathrm{cmol}^{+} \mathrm{kg}^{-1}$ de terre fine. Les valeurs indiquées en gras correspondent aux seuils définis par Bonneau (1980, dans Lévy, 1988 ) en dessous desquels les sols peuvent être considérés comme pauvres vis-à-vis de l'élément considéré. 
Tableau IV. Valeurs moyennes des différents éléments foliaires analysés.

\begin{tabular}{lccccc} 
- $\begin{array}{c}\text { Moyenne } \\
( \pm \text { écart type) }\end{array}$ & Minimum & Maximum & $\begin{array}{c}\text { Valeur optimale } \\
\text { (Bonneau, 1988) }\end{array}$ & $\begin{array}{c}\text { Valeur critique } \\
\text { (Bonneau, 1988) }\end{array}$ \\
\hline $\mathrm{N}$ & $1,59( \pm 0,17)$ & 1,24 & 2,06 & 1,2 à 1,5 & 0,8 à 1,0 \\
$\mathrm{P}$ & $0,12( \pm 0,02)$ & 0,088 & 0,178 & 0,12 à 0,15 & 0,08 à 0,1 \\
$\mathrm{Ca}$ & $0,19( \pm 0,06)$ & 0,08 & 0,31 & 0,10 à 0,12 & \\
$\mathrm{Mg}$ & $0,12( \pm 0,02)$ & 0,07 & 0,19 & & 0,2 à 0,3 \\
$\mathrm{~K}$ & $0,59( \pm 0,07)$ & 0,46 & 0,88 & 0,5 & \\
$\mathrm{~N} / \mathrm{P}$ & $13,4( \pm 2,4)$ & 8,55 & 22 & 14 &
\end{tabular}

Les teneurs sont exprimées en $\%$, et le rapport N/P est sans unité ( $n_{p}=120$ placettes $; n_{a}=600$ arbres). Selon Prévôt et Ollagnier (1956), le niveau critique est la concentration de l'élément pour laquelle la croissance n'atteint que $90 \%$ de la croissance maximale.

Tableau V. Matrice des coefficients de corrélation entre les concentrations foliaires et les valeurs des variables chimiques édaphiques.

\begin{tabular}{|c|c|c|c|c|c|c|}
\hline & \multicolumn{6}{|c|}{ Éléments foliaires } \\
\hline & $N$ & $P$ & $\mathrm{Ca}$ & $M g$ & $K$ & $N / P$ \\
\hline \multicolumn{7}{|c|}{ Éléments foliaires } \\
\hline $\mathrm{N}$ & 1 & & & & & \\
\hline $\mathrm{P}$ & 0,213 & 1 & & & & \\
\hline $\mathrm{Ca}$ & & 0,21 & 1 & & & \\
\hline $\mathrm{Mg}$ & & 0,369 & 0,396 & 1 & & \\
\hline $\mathrm{K}$ & & & $-0,409$ & $-0,195$ & 1 & \\
\hline $\mathrm{N} / \mathrm{P}$ & 0,417 & $-0,783$ & $-0,233$ & $-0,32$ & & 1 \\
\hline \multicolumn{7}{|c|}{ Horizon Al } \\
\hline \multicolumn{7}{|l|}{$N$} \\
\hline MO & & & $-0,417$ & & 0,278 & \\
\hline $\mathrm{C} / \mathrm{N}$ & & & $-0,27$ & & & \\
\hline $\mathrm{Ca}$ & & & 0,208 & 0,212 & $-0,195$ & $-0,228$ \\
\hline \multicolumn{7}{|l|}{$\mathrm{Mg}$} \\
\hline \multicolumn{7}{|l|}{$\mathrm{K}$} \\
\hline $\mathrm{S} / \mathrm{T}$ & & 0,244 & 0,502 & 0,322 & $-0,291$ & $-0,21$ \\
\hline \multicolumn{7}{|c|}{ Horizon minéral } \\
\hline $\mathrm{P}_{2} \mathrm{O}_{5}$ & & 0,522 & & & & $-0,412$ \\
\hline $\mathrm{Ca}$ & & & 0,23 & & & \\
\hline \multicolumn{7}{|l|}{$\mathrm{Mg}$} \\
\hline \multicolumn{7}{|l|}{$\mathrm{K}$} \\
\hline $\mathrm{S} / \mathrm{T}$ & & 0,269 & 0,438 & 0,195 & $-0,251$ & $-0,256$ \\
\hline
\end{tabular}

Seules les corrélations significatives au seuil de $5 \%$ sont présentées $\left(n_{p}=120\right.$ placettes). MO : matière organique. 
dans certains peuplements, contrairement au potassium pour lequel le niveau foliaire est optimal malgré la pauvreté du sol en cet élément. Pour les autres éléments, les teneurs observées correspondent à des valeurs optimales, ce qui confirme les très bonnes disponibilités au niveau du sol.

\section{Rôle de la nutrition minérale sur la croissance radiale}

La disponibilité du sol en phosphore ainsi que la nutrition en cet élément étant les facteurs les plus variables dans le contexte pédoclimatique de la région Pays-de-laLoire, nous avons dans un premier temps analysé la réponse des peuplements à cet élément.

La stratification des placettes selon la disponibilité en phosphore au niveau du premier horizon minéral montre un effet posi- tif de cet élément sur la croissance radiale des arbres pour des teneurs édaphiques supérieures à $0,07 \mathrm{~g} \cdot \mathrm{kg}^{-1}$ (fig 2). Cependant, pour la période 1983-1990, les écarts entre les accroissements des deux populations d'arbres n'apparaissent plus différents. On peut émettre l'hypothèse que les déficits hydriques répétés pendant cette période (fig 1) aient limité l'effet favorable du phosphore et ralenti notablement la croissance de ces arbres. L'hypothèse climatique semble d'ailleurs en grande partie confirmée par le comportement des arbres en 1991. En effet, le retour de meilleures conditions pluviométriques à cette date (fig 1) se traduit par une reprise significative de croissance des arbres bien alimentés en phosphore, alors que la croissance des autres peuplements continue de chuter.

L'effet du phosphore sur la croissance est confirmé quand on considère le niveau de la nutrition foliaire. Si l'on stratifie l'échan-

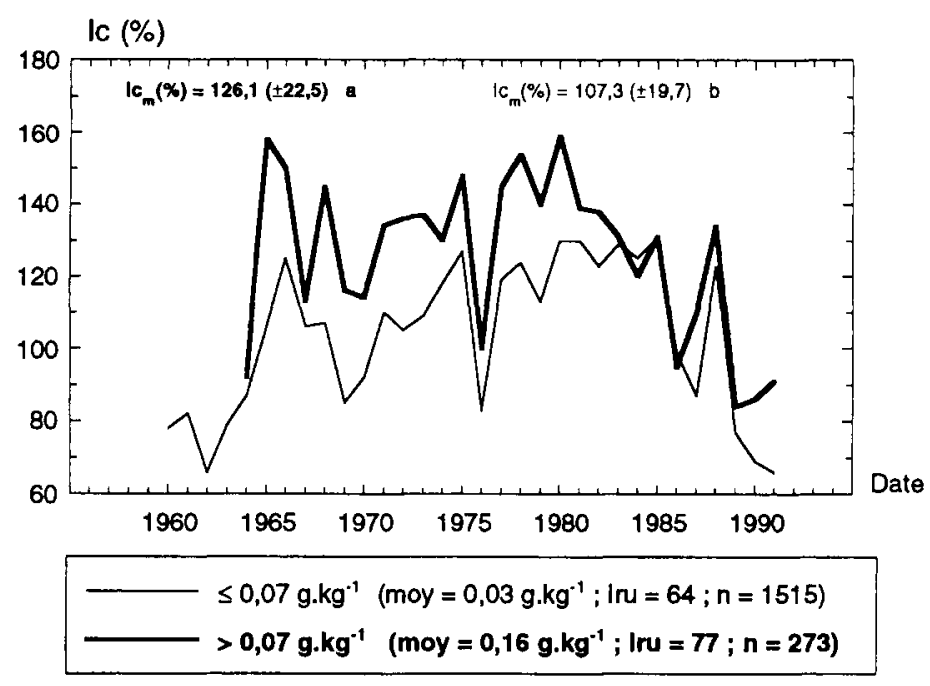

Fig 2. Évolution dans le temps de l'indice moyen de croissance radiale (Ic \%) selon la teneur en phosphore $\left(\mathrm{g} \cdot \mathrm{kg}^{-1}\right)$ du premier horizon minéral $(-20 /-30 \mathrm{~cm})($ moy $=$ valeur moyenne du paramètre pour la strate correspondante ; Iru = indice moyen de réserve utile ; $n=$ effectif d'arbres). Pour la comparaison des indices moyens de croissance radiale $\left(\mathrm{Ic}_{m}(\%)\right)$, des lettres toutes différentes indiquent des différences significatives au seuil de $5 \%$ (analyse de variance suivie du test $\mathrm{F}$ de Fischer sur la période 1964-1991). 
tillon initial selon la teneur des aiguilles en phosphore (P) ou le rapport foliaire N/P, trois populations d'arbres s'individualisent nettement (fig 3). Si l'on considère les variations du rapport $\mathrm{N} / \mathrm{P}$, la croissance la plus importante correspond à une valeur moyenne de l'ordre de 10 , ce qui équivaut à des teneurs foliaires moyennes en azote et phosphore respectivement de $1,49 \%$ et $0,15 \%$, et à une disponibilité en phosphore au niveau du premier horizon minéral de l'ordre de $0,09 \mathrm{~g} \cdot \mathrm{kg}^{-1}$ (tableau VI). Le niveau moyen de croissance correspond à un rapport de l'ordre de 14, pour une teneur en azote foliaire de 1,60\% et, pour le phosphore, de $0,12 \%$ et $0,05 \mathrm{~g} \cdot \mathrm{kg}^{-1}$ respectivement pour les feuilles et le sol. Enfin, la croissance la plus faible est observée pour un rapport de l'ordre de 18 correspondant à des valeurs moyennes de $1,74 \%$ et $0,10 \%$ pour l'azote et le phosphore, et une disponibilité en phosphore réduite autour de $0,03 \mathrm{~g} \cdot \mathrm{kg}^{-1}$. Cependant pour cette dernière strate, le faible niveau de croissance radiale observé

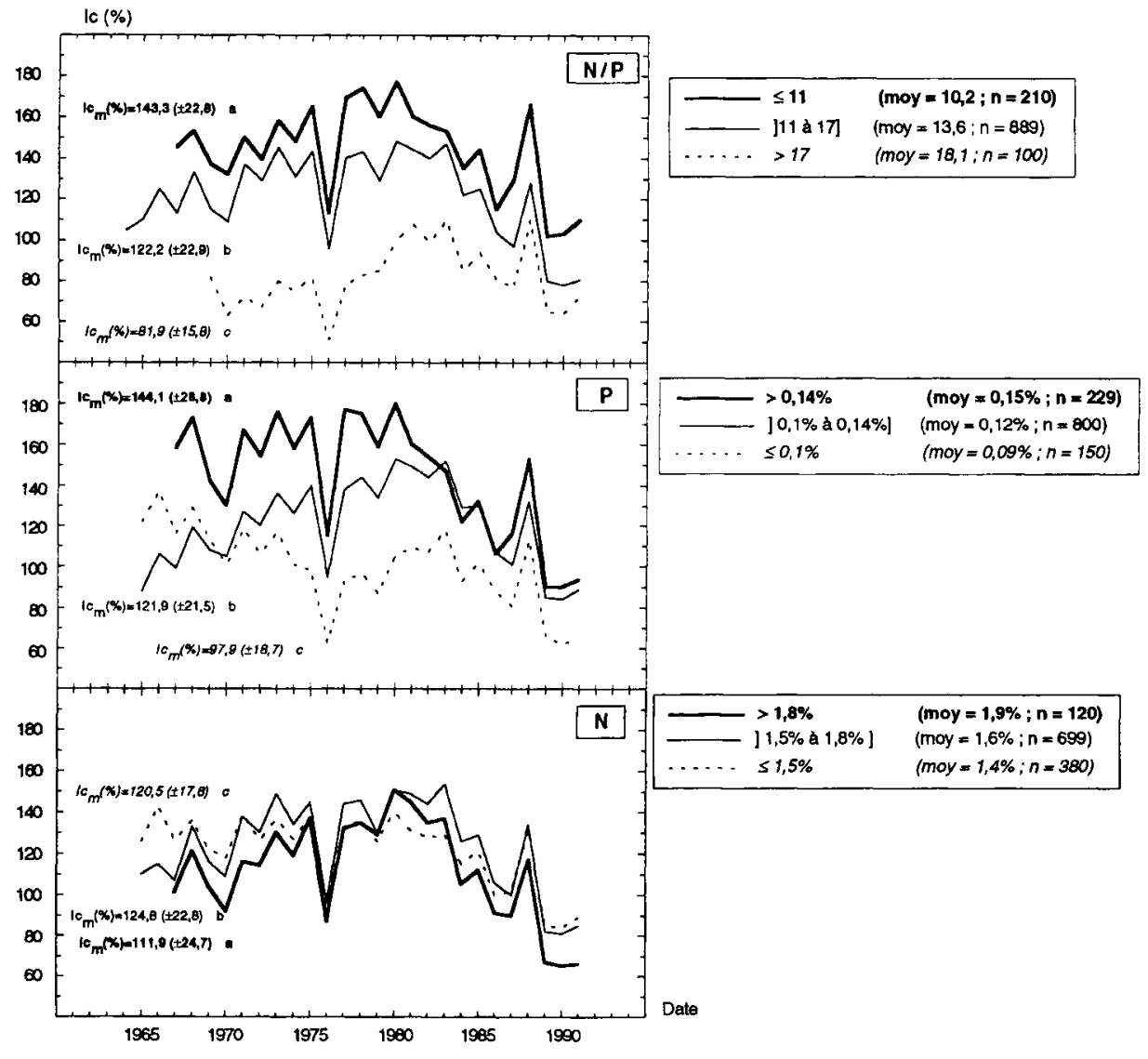

Fig 3. Évolution dans le temps de l'indice moyen de croissance radiale (Ic \%) selon le rapport foliaire $\mathrm{N} / \mathrm{P}$, la teneur en phosphore $(\mathrm{P})$ et en azote $(\mathrm{N})$ des aiguilles (moy = valeur moyenne du paramètre pour la strate correspondante ; $n=$ effectif d'arbres). Pour la comparaison des indices moyens de croissance radiale $\left(\mathrm{Ic}_{m}(\%)\right)$, des lettres toutes différentes indiquent des différences significatives au seuil de $5 \%$ (analyse de variance suivie du test F de Fischer sur la période 1969-1991). 
Tableau VI. Valeurs moyennes ( \pm écart type) des différents paramètres chimiques foliaires et édaphiques pour les différentes classes de la variable N/P distinguées.

\begin{tabular}{|c|c|c|c|c|c|}
\hline & \multicolumn{5}{|c|}{ Analyses foliaires (\%) } \\
\hline & $N$ & $P$ & $\mathrm{Ca}$ & $M g$ & $K$ \\
\hline $\begin{array}{l}\mathrm{N} / \mathrm{P} \leq 11 \\
\quad(\mathrm{moy}=10,2)\end{array}$ & $\begin{array}{c}1,49( \pm 0,15) \\
a\end{array}$ & $\begin{array}{c}0,15( \pm 0,014) \\
a\end{array}$ & $\begin{array}{c}0,22( \pm 0,06) \\
a\end{array}$ & $\begin{array}{c}0,13( \pm 0,017) \\
\mathrm{a}\end{array}$ & $\begin{array}{c}0,58( \pm 0,08) \\
\mathrm{a}\end{array}$ \\
\hline $\begin{array}{c}\mathrm{N} / \mathrm{P}=] 11 \text { à } 17] \\
(\mathrm{moy}=13,6)\end{array}$ & $\begin{array}{c}1,60( \pm 0,16) \\
b\end{array}$ & $\begin{array}{c}0,12( \pm 0,017) \\
\text { b }\end{array}$ & $\begin{array}{c}0,18( \pm 0,05) \\
b\end{array}$ & $\begin{array}{c}0,12( \pm 0,018) \\
b\end{array}$ & $\begin{array}{c}0,59( \pm 0,07) \\
a\end{array}$ \\
\hline \multirow[t]{3}{*}{$\begin{array}{l}\text { N/P }>17 \\
\quad(\operatorname{moy}=18,1)\end{array}$} & $\begin{array}{c}1,74( \pm 0,19) \\
c\end{array}$ & $\begin{array}{c}0,10( \pm 0,007) \\
c\end{array}$ & $\begin{array}{c}0,17( \pm 0,04) \\
b\end{array}$ & $\begin{array}{c}0,11( \pm 0,010) \\
b\end{array}$ & $\begin{array}{c}0,59( \pm 0,06) \\
\mathrm{a}\end{array}$ \\
\hline & \multirow{2}{*}{$\begin{array}{c}\text { Horizon Al } \\
S / T\end{array}$} & \multicolumn{2}{|c|}{ Horizon minéral } & Indice de & \\
\hline & & $S / T$ & $P_{2} O_{5}\left(g \cdot k g^{-1}\right)$ & & \\
\hline $\begin{array}{l}\mathrm{N} / \mathrm{P} \leq 11 \\
\quad(\operatorname{moy}=10,2)\end{array}$ & $\begin{array}{c}56( \pm 30) \\
a\end{array}$ & $\begin{array}{c}39( \pm 34) \\
a\end{array}$ & $\begin{array}{c}0,09( \pm 0,09) \\
\mathrm{a}\end{array}$ & $\begin{array}{c}74( \pm 61) \\
a\end{array}$ & \\
\hline $\begin{array}{c}\mathrm{N} / \mathrm{P}=] 11 \text { à } 17] \\
(\operatorname{moy}=13,6)\end{array}$ & $\begin{array}{c}40( \pm 22) \\
b\end{array}$ & $\begin{array}{c}22( \pm 21) \\
b\end{array}$ & $\begin{array}{c}0,05( \pm 0,04) \\
b\end{array}$ & $\begin{array}{c}74( \pm 47) \\
a\end{array}$ & \\
\hline $\begin{array}{l}\mathrm{N} / \mathrm{P}>17 \\
\quad(\operatorname{moy}=18,1)\end{array}$ & $\begin{array}{c}40( \pm 24) \\
b\end{array}$ & $\begin{array}{c}26( \pm 26) \\
b\end{array}$ & $\begin{array}{c}0,03( \pm 0,02) \\
b\end{array}$ & $\begin{array}{c}46( \pm 24) \\
b\end{array}$ & \\
\hline
\end{tabular}

Des lettres toutes différentes indiquent une différence significative au seuil de $5 \%$ (analyse de variance suivie du test $\mathrm{F}$ de Fischer).

semble être également la résultante de conditions d'approvisionnement en eau limitantes qui s'expriment à travers un indice moyen de réserve utile nettement inférieur à celui des autres peuplements (tableau VI).

Comme précédemment, on observe pour les différentes courbes des variations interannuelles plus ou moins marquées, et une diminution assez régulière de la croissance radiale pour la période sèche 1983-1990.

La stratification selon le niveau de la nutrition azotée ne permet pas de discriminer aussi nettement que précédemment des niveaux de croissance différents. Quels que soient les seuils considérés, les courbes se chevauchent sans jamais se séparer systématiquement (fig 3). Cependant, si l'on considère les indices moyens de croissance, on constate que le niveau de croissance le plus faible est associé à la plus forte teneur en azote $(>1,8 \%)$. En fait, contrairement au phosphore qui, considéré isolément, per- met de discriminer nettement des niveaux de croissance radiale différents, l'azote ne joue pas un rôle direct (pas de teneurs limitantes), mais influence négativement la croissance à travers le rapport N/P qui augmente pour les peuplements où la teneur en azote est élevée (tableau VII). Le déséquilibre ne semble effectivement pas lié au phosphore, car la valeur observée pour les arbres de ces peuplements est équivalente, voire légèrement supérieure, à celle des autres peuplements. Pour les deux autres populations d'arbres, les différences de croissance ne semblent pas être en relation avec la qualité de la nutrition. Il est possible que ce soit la différence des conditions d'approvisionnement en eau (Iru) qui explique partiellement ces comportements (bien que celle-ci n'apparaisse pas significative au niveau statistique).

Pour les autres éléments ( $\mathrm{Ca}, \mathrm{Mg}$ et $\mathrm{K}$ ), les différences entre les populations d'arbres 
Tableau VII. Valeurs moyennes ( \pm écart type) de différents paramètres foliaires et édaphiques pour les différents niveaux de nutrition azotée distingués.

\begin{tabular}{|c|c|c|c|c|c|}
\hline & \multicolumn{5}{|c|}{ Analyses foliaires (\%) } \\
\hline & $P$ & $N / P$ & $\mathrm{Ca}$ & $M g$ & $K$ \\
\hline \multirow{6}{*}{$\begin{array}{l}\mathrm{N}<1,5 \% \\
(\operatorname{moy}=1,4 \%) \\
\mathrm{N}=11,5 \% \text { à } 1,8 \%] \\
(\operatorname{moy}=1,6 \%) \\
\mathrm{N}>1,8 \% \\
(\operatorname{moy}=1,9 \%)\end{array}$} & $\begin{array}{c}0,12( \pm 0,012) \\
a\end{array}$ & $\begin{array}{c}12( \pm 1,9) \\
a\end{array}$ & $\begin{array}{c}0,19( \pm 0,06) \\
a\end{array}$ & $\begin{array}{c}0,12( \pm 0,02) \\
\mathrm{a}\end{array}$ & $\begin{array}{c}0,58( \pm 0,06) \\
a\end{array}$ \\
\hline & $0,12( \pm 0,019)$ & $13,8( \pm 2)$ & $0,19( \pm 0,06)$ & $0,12( \pm 0,02)$ & $0,58( \pm 0,07)$ \\
\hline & $\begin{array}{c}a b \\
0,13( \pm 0,028)\end{array}$ & $\begin{array}{c}b \\
15,2( \pm 3.6)\end{array}$ & $\frac{a}{0,17( \pm 0,04)}$ & $\frac{a}{0,12}( \pm 0,02)$ & $\begin{array}{c}a \\
0,62( \pm 0,04)\end{array}$ \\
\hline & $b$ & $\mathrm{c}$ & a & a & $\mathrm{a}$ \\
\hline & \multirow{2}{*}{$\begin{array}{l}\text { Horizon Al } \\
\quad S / T\end{array}$} & \multicolumn{2}{|c|}{ Horizon minéral } & \multirow{2}{*}{$\begin{array}{l}\text { Indice de } \\
\text { résenve utile }\end{array}$} & \\
\hline & & $S / T$ & $P_{2} O_{5}\left(g \cdot k g^{-l}\right)$ & & \\
\hline $\begin{array}{l}\mathrm{N}<1,5 \% \\
(\mathrm{moy}=1.4 \%)\end{array}$ & $\begin{array}{c}44( \pm 28) \\
a\end{array}$ & $\begin{array}{c}27( \pm 29) \\
a\end{array}$ & $\begin{array}{c}0,06( \pm 0,06) \\
\mathrm{ab}\end{array}$ & $\begin{array}{c}65( \pm 44) \\
a\end{array}$ & \\
\hline $\begin{array}{c}\mathrm{N}=] 1,5 \% \text { à } 1,8 \%] \\
(\operatorname{moy}=1,6 \%)\end{array}$ & $\begin{array}{c}43( \pm 23) \\
b\end{array}$ & $\begin{array}{c}27( \pm 24) \\
a\end{array}$ & $\begin{array}{c}0,05( \pm 0,04) \\
\mathrm{a}\end{array}$ & $\begin{array}{c}75( \pm 48) \\
\mathrm{a}\end{array}$ & \\
\hline $\begin{array}{l}\mathrm{N}>1,8 \% \\
\quad(\operatorname{moy}=1,9 \%)\end{array}$ & $\begin{array}{c}44( \pm 22) \\
b\end{array}$ & $\begin{array}{c}22( \pm 19) \\
a\end{array}$ & $\begin{array}{c}0,08( \pm 0,07) \\
b\end{array}$ & $\begin{array}{c}76( \pm 70) \\
b\end{array}$ & \\
\hline
\end{tabular}

Des lettres toutes différentes indiquent une différence significative au seuil de $5 \%$ (analyse de variance suivie du test $F$ de Fischer).

sont peu importantes, et les valeurs moyennes mesurées égalent ou dépassent le niveau des seuils optimaux (tableaux VI et VII). De plus, quels que soient les seuils considérés, les stratifications effectuées à partir des teneurs foliaires ou édaphiques n'ont pas permis d'individualiser significativement des populations d'arbres. Ainsi dans le secteur ligérien étudié, seule la nutrition phosphorée apparaît être effectivement limitante pour la croissance radiale.

\section{Rôle de l'approvisionnement local en eau}

Quel que soit l'indice d'humidité du milieu utilisé, la stratification aboutit toujours à la séparation des placettes les plus « sèches » des placettes les plus «humides» (fig 4). Les placettes les plus sèches sont caractérisées par des faibles valeurs d'indices de réserve utile ou des conditions d'apports latéraux d'eau défavorables ou des sols superficiels. En fait, même si ces deux derniers paramètres permettent de discriminer nettement des niveaux de croissance radiale différents, l'augmentation de la profondeur du sol et l'amélioration des conditions de drainage coïncident avec une augmentation de l'indice de réserve utile. Il est donc impossible de distinguer clairement l'importance relative sur la croissance de chaque indice de la qualité de l'approvisionnement en eau.

Si l'on considère la stratification selon l'indice de réserve utile, la séparation des courbes coïncide avec des crises de croissance, variables en durée et en intensités, concomitantes aux principaux déficits pluviométriques de ces dernières années. Ainsi, par rapport aux stations «humides " (Iru > 75), la croissance des arbres des stations " sèches " (Iru $\leq 25)$ s'individualise après les déficits des années 1958-1962, et 


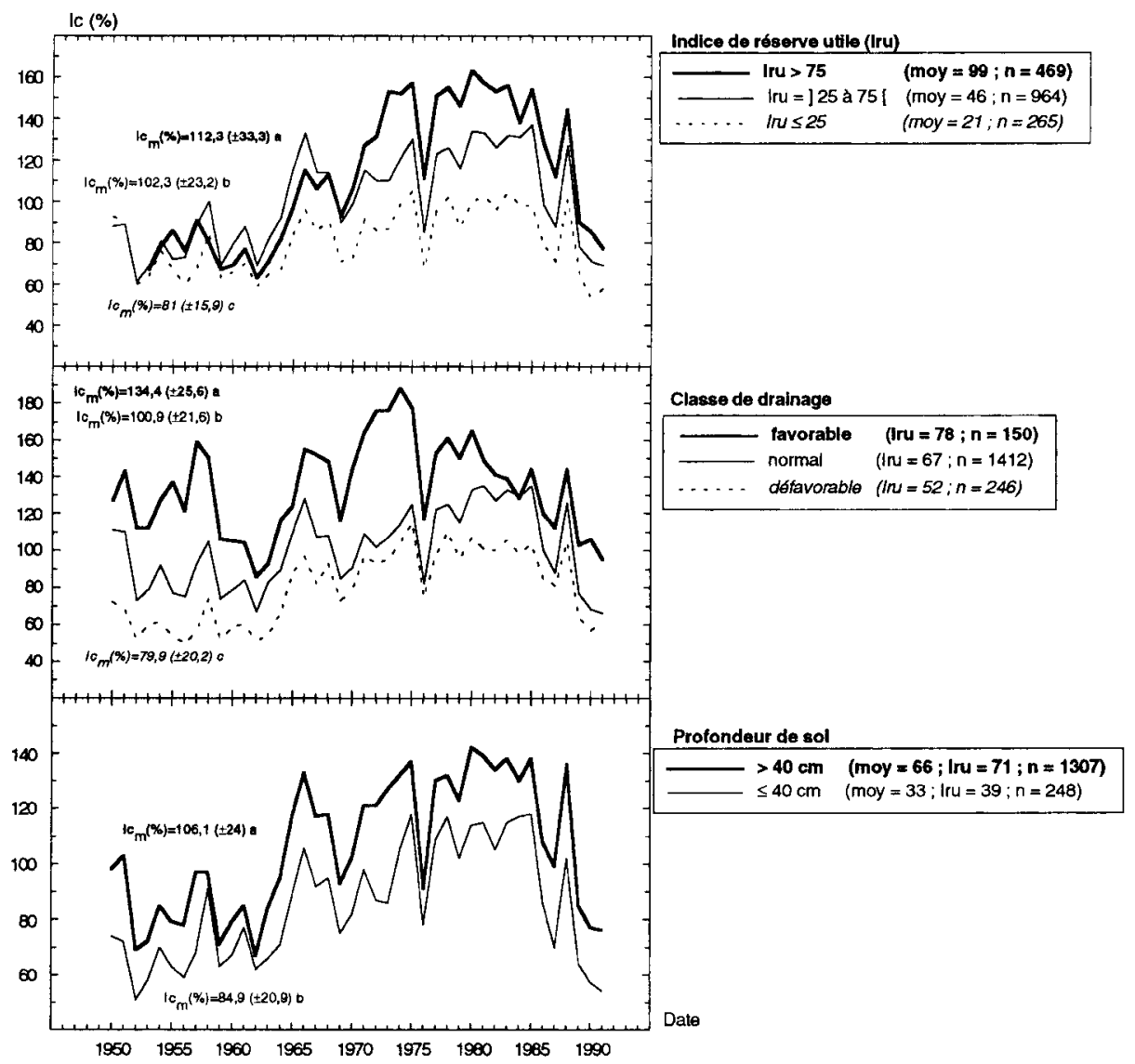

Fig 4. Évolution dans le temps de l'indice moyen de croissance radiale (Ic \%) selon l'indice de réserve utile (Iru), la classe de drainage et la profondeur du sol $(\mathrm{en} \mathrm{cm})$. moy = valeur moyenne du paramètre pour la strate correspondante ; Iru = indice moyen de réserve utile ; $n=$ effectif d'arbres. Pour la comparaison des indices moyens de croissance radiale $\left(\mathrm{Ic}_{m}(\%)\right)$, des lettres toutes différentes indiquent des différences significatives au seuil de $5 \%$ (analyse de variance suivie du test $\mathrm{F}$ de Fischer sur la période 1953-1991).

celle des stations « moyennes » (Iru =] 25 à 75]) après la sécheresse de 1969. Après 1976, les écarts augmentent, et seules les années exceptionnellement sèches 1989-1990 réduisent en partie les différences. Ainsi, la réserve utile en eau du sol semble avoir « prédisposé » les peuplements aux effets des sécheresses. Nous reviendrons sur ce point dans la discussion.

\section{Interaction nutrition phosphorée et approvisionnement en eau}

Face à l'importance des effets de la nutrition en phosphore et de l'approvisionnement en eau sur la croissance radiale du pin laricio, nous avons cherché à analyser plus finement le comportement des arbres visà-vis du facteur hydrique dans le cas d'une 


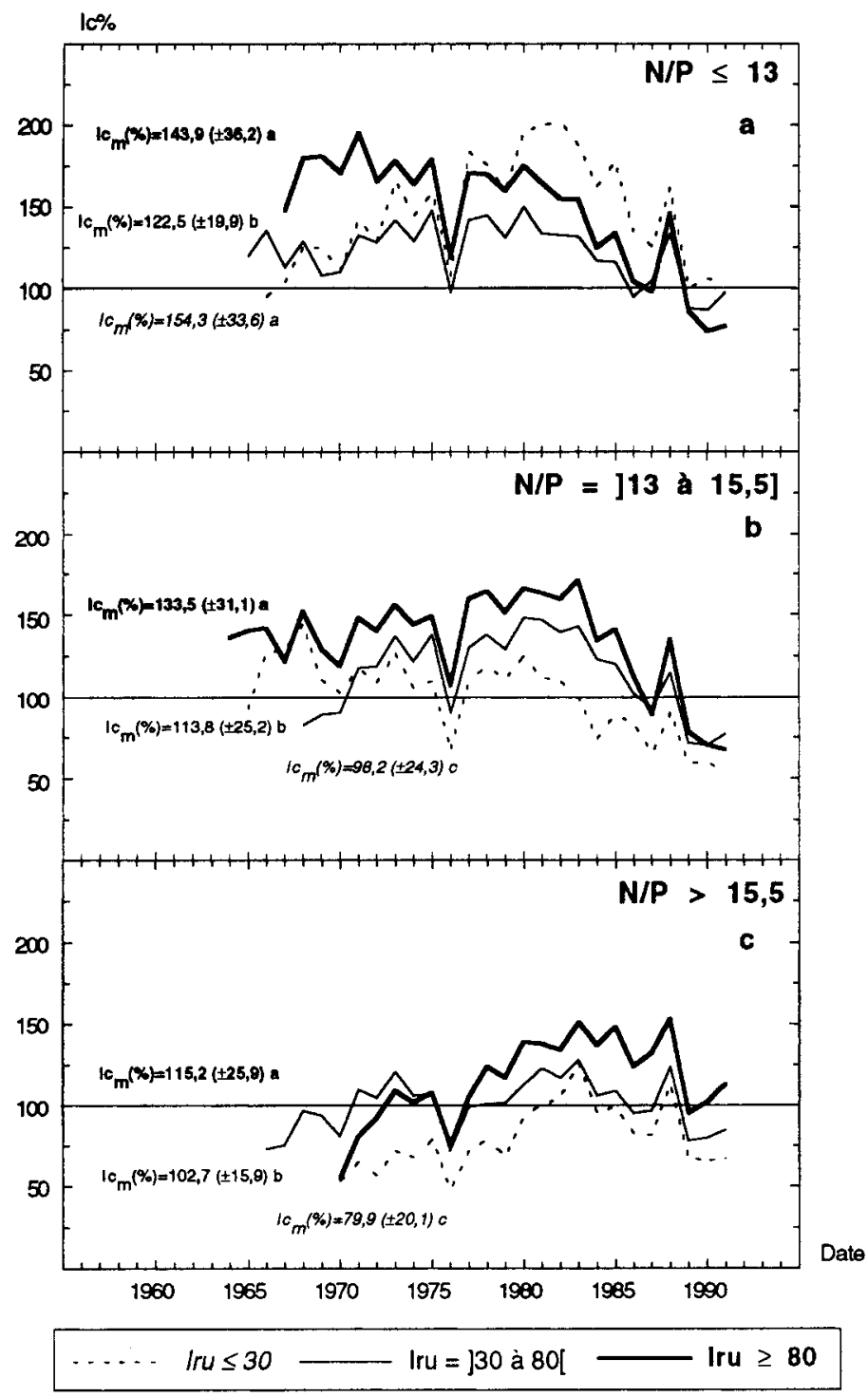

Fig 5. Évolution dans le temps de l'indice moyen de croissance radiale (Ic \%) selon l'indice de réserve utile pour trois niveaux de nutrition phosphorée. Pour la comparaison des indices moyens de croissance radiale $\left(\mathrm{Ic}_{m}(\%)\right)$, des lettres toutes différentes indiquent des différences significatives au seuil de $5 \%$ (analyse de variance suivie du test F de Fischer sur la période 1970-1991). Les effectifs de chaque strate sont présentés dans le tableau VIII. 
nutrition « optimale » $(\mathrm{N} / \mathrm{P} \leq 13)$, « critique » $(\mathrm{N} / \mathrm{P}=] 13$ à 15,5$])$ et « déficiente » $(\mathrm{N} / \mathrm{P}>15,5)$. Les résultats sont présentés à la figure 5 et au tableau VIII.

Si l'on considère le niveau moyen de croissance radiale $\left(\mathrm{Ic}_{m} \%\right)$, on constate d'une part, que les niveaux de croissance les plus élevés sont associés aux meilleures conditions de nutrition phosphorée (fig 5a), et d'autre part, que l'augmentation de la réserve utile du sol permet de discriminer, dans la majorité des cas, des niveaux de croissance différents (fig $5 b$ et $5 \mathrm{c}$ ). La croissance la plus forte est alors associée aux meilleures conditions d'alimentation hydrique ( $\mathrm{Iru} \geq 80$ ).
Cependant, cette relation ne semble pas toujours vérifiée. En effet, si l'on considère seulement le cas des peuplements dont la nutrition en phosphore est optimale (fig 5a), on constate que la croissance moyenne $\left(\mathrm{Ic}_{m} \%\right)$ des arbres à faible réserve utile (Iru $\leq 30$ ) est équivalente à celle des arbres à forte réserve utile (Iru $\geq 80$ ). Pour ces peuplements, tout se passe comme si la très faible disponibilité en eau du sol n'avait globalement aucun effet limitant sur la croissance! Ce comportement particulier trouve en partie son explication dans la structure actuelle et les niveaux de compétition observés dans ces peuplements. En effet, l'analyse des facteurs d'élancement montre que ces

Tableau VIII. Valeurs moyennes ( \pm écart type) de l'indice de réserve utile (Iru), du rapport foliaire $\mathrm{N} / \mathrm{P}$ et du facteur d'élancement des arbres H/D associées à chaque sous-population d'arbres définie suite aux stratifications croisées entre le niveau de la nutrition phosphorée et les conditions d'approvisionnement en eau.

\begin{tabular}{|c|c|c|c|c|c|}
\hline & \multicolumn{5}{|c|}{$N / P \leq 13$} \\
\hline & Iru & $N / P$ & $H / D$ & Effectif & $\hat{A} g e$ (ans) \\
\hline \multicolumn{6}{|l|}{ (a) } \\
\hline Iru $\leq 30$ & $24( \pm 3) a$ & $11,5( \pm 1,0) \mathrm{a}$ & $53( \pm 4) a$ & 60 & $26( \pm 2)$ \\
\hline Iru $=] 30$ à 80[ & $48( \pm 14) b$ & $11,3( \pm 1,2) \mathrm{a}$ & $61( \pm 9) b$ & 289 & $24( \pm 3)$ \\
\hline \multirow[t]{3}{*}{ Iru $\geq 80$} & $101( \pm 17) \mathrm{c}$ & $11,2( \pm 0,8) a$ & $59( \pm 4) c$ & 100 & $25( \pm 3)$ \\
\hline & \multicolumn{5}{|c|}{$N / P=1 / 3$ à 15,5$]$} \\
\hline & Iru & $N / P$ & $H / D$ & Effectif & Âge (ans) \\
\hline \multicolumn{6}{|l|}{ (b) } \\
\hline Iru $\leq 30$ & $23( \pm 6) \mathrm{a}$ & $14,4( \pm 0,9) \mathrm{a}$ & $64( \pm 8) a$ & 60 & $28( \pm 3)$ \\
\hline Iru $=] 30$ à 80[ & $51( \pm 12) b$ & $14,2( \pm 0,9) a$ & $62( \pm 6) a$ & 210 & $24( \pm 3)$ \\
\hline \multirow[t]{3}{*}{ Iru $\geq 80$} & $101( \pm 23) c$ & $14,4( \pm 0,8)$ a & $59( \pm 6) b$ & 190 & $25( \pm 4)$ \\
\hline & \multicolumn{5}{|c|}{$N / P>15.5$} \\
\hline & Iru & $N / P$ & $H / D$ & Effectif & $\hat{A} g e$ (ans) \\
\hline \multicolumn{6}{|l|}{ (c) } \\
\hline Iru $\leq 30$ & $25( \pm 5) \mathrm{a}$ & $16,9( \pm 0,6) a$ & $56( \pm 4) a$ & 60 & $22( \pm 4)$ \\
\hline Iru $=] 30$ à 80[ & $54( \pm 13) b$ & $17,5( \pm 2) b$ & $55( \pm 8)$ a & 100 & $24( \pm 4)$ \\
\hline Iru $\geq 80$ & $89( \pm 11) \mathrm{c}$ & $16,4( \pm 0,8) \mathrm{c}$ & $56( \pm 4) a$ & 50 & $23( \pm 4)$ \\
\hline
\end{tabular}

(a), (b), (c) correspondent aux graphes de la figure 5. Des lettres toutes différentes faisant suite aux valeurs moyennes indiquent une différence significative au seuil de $5 \%$ (analyse de variance suivie du test F de Fischer). 
arbres ont été, en moyenne, moins concurrencés que les autres (H/D plus bas) (tableau VIII). L'origine de cette moindre concurrence n'est pas évidente. Elle peut provenir d'une densité initiale de plantation plus faible, de pratique plus régulière d'éclaircies... Cependant, au vu de la dynamique d'accroissement de ces arbres (fig 5a ; trait pointillé), qui devient nettement différente des autres arbres après 1976, on peut également émettre l'hypothèse que la forte sécheresse ait entraîné des mortalités importantes au sein de ces peuplements, et que par la suite, les arbres ayant survécu à cette crise aient bénéficié de plus d'espace et d'une moindre compétition pour l'eau. Étant moins concurrencés, ils ont alors exprimé pleinement leur potentiel de croissance, et ont mieux résisté aux autres crises, notamment à celle qui a débuté en 1983, et dont les effets sont particulièrement visibles sur le niveau de croissance des arbres bénéficiant de conditions hydriques et minérales optimales, mais subissant des niveaux moyens de concurrence plus forts (arbres plus vigoureux ; H/D plus élevés) (tableau VIII). Nous reviendrons sur ces points dans la discussion.

L'année 1976 semble être une date charnière car, dans la majorité des cas, les niveaux d'accroissement s'individualisent nettement et systématiquement selon le gradient d'indice de réserve utile après cette date. Cette observation tend à confirmer le rôle décisif du climat, et particulièrement de la pluviométrie, sur certains comportements observés actuellement, et également le rôle prédisposant de la réserve utile en eau du sol.

\section{DISCUSSION ET CONCLUSIONS}

Cet article avait pour objectif d'étudier le rôle de la nutrition minérale et des conditions d'approvisionnement local en eau sur la croissance radiale des arbres de peuplements adultes de pin laricio de Corse dans
l'Ouest de la France (région Pays-de-laLoire).

En ce qui concerne la nutrition minérale, notre étude montre qu'il existe une relation étroite entre la qualité de la nutrition en phosphore et le niveau d'accroissement radial (fig 3). Ce résultat confirme les observations faites par différents auteurs sur le rôle majeur du phosphore pour la croissance du pin laricio (Bonneau, 1970 ; Miller et Miller, 1976 ; Miller et al, 1980, 1981 ; Moffat et al, 1991), et coïncide avec les résultats observés pour d'autres résineux comme Pinus radiata (Mesanza et al, 1993 ; Raupach et al, 1969), Pinus taeda et Pinus eliottii (McNeil et al, 1984 ; Valentine et Allen, 1990), Pinus pinaster (Lemoine, 1993). Une telle relation est assez générale, car cet élément intervient dans de multiples réactions physiologiques, et notamment l'assimilation carbonée (Miller, 1986 ; Sheriff et al, 1986 ; Agrawal, 1988).

En ce qui concerne les seuils de fertilité minérale des sols et les normes de composition foliaire pour le pin laricio, nos résultats divergent partiellement des valeurs moyennes couramment admises (Bonneau, 1988 ; Lévy, 1988). Pour le potassium, la faible disponibilité apparente des sols ne se traduit pas par une nutrition déficiente (teneur foliaire optimale de $0,59 \%$ ), ni par une réduction significative de la croissance radiale. Ainsi, selon nos résultats, les seuils en dessous desquels les sols peuvent être considérés comme peu fertiles pour le potassium doivent se situer en dessous de $0,2 \mathrm{cmol}+\mathrm{kg}^{-1}$ pour l'horizon $\mathrm{A}_{1}$ (au lieu de $0,6 \mathrm{cmol}+\mathrm{kg}^{-1}$ ) et de $0,08 \mathrm{cmol}+\mathrm{kg}^{-1}$ pour le premier horizon minéral (au lieu de $0,12 \mathrm{cmol}+\mathrm{kg}^{-1}$ ) (tableau III).

Pour le phosphore, qui est l'élément minéral limitant essentiel dans le contexte ligérien étudié, les valeurs de teneurs foliaires en deçà desquelles la réduction de croissance est nette sont supérieures à celles données par Bonneau (1988). Alors que cet auteur donne comme teneurs optimales 
(c'est-à-dire teneurs associées à la croissance la plus forte) des valeurs comprises entre $0,12 \%$ et $0,15 \%$, et comme valeurs critiques des teneurs de 0,08 à $0,10 \%$ (tableau IV), nos résultats montrent qu'une valeur de $0,12 \%$ entraîne déjà une réduction significative de croissance, que la valeur de $0,10 \%$ se traduit par une croissance très réduite, et que seule la valeur de $0,15 \%$ est associée au niveau de croissance le plus élevé. Parallèlement, pour les rapports foliaires N/P, la valeur de 14 (généralement considérée comme optimale) se traduit déjà par une moindre croissance ; la croissance la plus faible et la plus élevée étant respectivement associée à des valeurs de 18 et 10 (fig 3 et tableau VI).

Pour la nutrition azotée, nos résultats montrent qu'elle n'agit pas directement sur la croissance des arbres (pas de valeurs limitantes), mais à travers une accentuation du déséquilibre N/P (tableaux VI et VII). Cependant, nous ne pouvons pas conclure de façon certaine sur l'origine de l'augmentation de la nutrition azotée observée dans certains peuplements. Dans un cas (tableau VI), cette augmentation pourrait être induite par la carence en phosphore, les fortes teneurs en azote étant associées aux faibles teneurs en phosphore. Dans l'autre cas (tableau VII), celle-ci semble indépendante des autres facteurs nutritionnels, car les différents niveaux de nutrition azotée sont associés à des niveaux de nutrition phosphorée comparables.

Enfin, pour le calcium et le magnésium, nous n'avons pas pu apprécier leur rôle réel sur la croissance car les valeurs observées égalent ou dépassent les teneurs optimales.

L'ensemble de ces résultats permet donc de mieux cerner les exigences nutritionnelles du pin laricio de Corse, et ainsi de préciser la qualité de la nutrition foliaire associée à chaque niveau de croissance (tableau IX). En toute rigueur, les valeurs proposées ici ne doivent s'appliquer que pour l'Ouest de la France, et pour diagnostiquer d'éventuels problèmes nutritionnels dans des peuplements adultes (25-30 ans). En effet, les travaux de Miller et al (1981) et Miller (1986) ont montré que les exigences minérales du pin laricio de Corse varient avec l'âge. Selon ces auteurs, l'optimum de concentration en azote foliaire évolue de $3,3 \%$ chez des jeunes semis de 4 mois à

Tableau IX. Teneurs foliaires associées à chaque niveau de croissance radiale des peuplements adultes (25-30 ans) de pin laricio de Corse dans la région Pays-de-la-Loire.

\begin{tabular}{|c|c|c|c|}
\hline & $\begin{array}{c}\text { Teneurs associées } \\
\text { à la plus faible } \\
\text { croissance }\end{array}$ & $\begin{array}{c}\text { Teneurs associées } \\
\text { à la croissance } \\
\text { moyenne }\end{array}$ & $\begin{array}{c}\text { Teneurs associées } \\
\text { à la plus forte } \\
\text { croissance }\end{array}$ \\
\hline$N(\%)$ & & & $\geq 1,2(2,1)$ \\
\hline $\mathrm{P}(\%)$ & $\begin{array}{c}0,10 \\
(0,09 \text { à } 0,11)\end{array}$ & $\begin{array}{c}0,12 \\
(0,12 \grave{\mathrm{a}} 0,13)\end{array}$ & $\begin{array}{c}0,15 \\
(0,14 \text { à } 0,18)\end{array}$ \\
\hline $\mathrm{Ca}(\%)$ & & & $\geq 0,08(0,31)$ \\
\hline $\operatorname{Mg}(\%)$ & & & $\geq 0,07(0,16)$ \\
\hline $\mathrm{K}(\%)$ & & & $\geq 0,5(0,88)$ \\
\hline $\mathrm{N} / \mathrm{P}$ & $\begin{array}{c}18 \\
(17 \grave{a} 22)\end{array}$ & $\begin{array}{c}14 \\
(12 \text { à } 16)\end{array}$ & $\begin{array}{c}10 \\
(8 \text { à } 11)\end{array}$ \\
\hline
\end{tabular}

Pour N, Ca, Mget K, les chiffres entre parenthèses et en italique correspondent aux valeurs maximales mesurées. 
$1,5 \%$ chez des arbres de 2 à $2,5 \mathrm{~m}$ de hauteur (4 ans), et remonte à environ $2 \%$ chez des arbres adultes.

De plus, et principalement pour le phosphore, bien que chaque teneur foliaire soit associée à des niveaux de croissance différents, on ne peut pas affirmer de façon certaine que les valeurs extrêmes de $0,10 \%$ et $0,15 \%$ correspondent au véritable seuil de carence et à la teneur optimale en cet élément. En effet, une vraie carence en phosphore se traduit non seulement par une réduction notable de croissance, mais également par un rougissement caractéristique des aiguilles et une perte foliaire ; symptômes qui n'ont pas été non observés ici. Pour la teneur optimale, il est également possible que, compte tenu du génotype et du climat, le maximum de croissance réel soit associé à une teneur plus élevée. Pour tester cette hypothèse, il faudrait alors augmenter « artificiellement» la teneur en cet élément, et suivre parallèlement la croissance des arbres, ce qui dépasse le cadre de l'étude présentée ici.

La qualité de la nutrition phosphorée dépend en grande partie de la disponibilité de cet élément au niveau du sol (tableaux $\mathrm{V}$ et VI). Cependant, bien que la stratification selon la disponibilité édaphique en phosphore ait permis de discriminer des niveaux de croissance différents (fig 2), on peut penser que sur des peuplements plus âgés, cette discrimination serait moins forte ou tout du moins plus difficile à mettre en évidence. En effet, les travaux de Miller et al (1981) et Ranger (1981) sur le pin laricio de Corse, et ceux de Le Goaster et al (1991) et Dambrine et al (1991) sur l'épicéa commun ont montré que, quand les peuplements vieillissent, l'intensité des prélèvements au niveau du sol décroît parallèlement à l'augmentation des transferts internes. Pour le pin laricio, les transferts internes des parties âgées vers les organes en croissance représentent entre 30 et $65 \%$ de la quantité totale de bioéléments contenue dans les tissus de l'année. Pour l'épicéa commun, les peuplements sont totalement autonomes vis-à-vis du prélèvement racinaire de phosphore destiné à l'accroissement de biomasse ligneuse du tronc dès l'âge de 30 ans (translocation en provenance des cernes plus âgés). Ce phénomène représente donc une économie naturelle très importante d'éléments nutritifs, et pourrait expliquer en partie la frugalité de certains peuplements forestiers adultes qui poussent sur des sols souvent pauvres (Nambiar et Fife, 1991 ; Helmisaari, 1992). Il pourrait également expliquer en partie l'absence de corrélation entre la nutrition foliaire en azote et les variables chimiques édaphiques (tableau V).

Quoi qu'il en soit, dans le contexte de l'étude, le diagnostic foliaire, en détectant les anomalies d'alimentation minérale, apparaît être un moyen fiable et plus précis que les analyses du sol, qui sont néanmoins complémentaires, pour évaluer le statut nutritionnel actuel et passé des peuplements et discriminer les niveaux de croissance des arbres.

La croissance radiale est également dépendante de la quantité d'eau disponible dans le sol. Ainsi, il est possible de différencier les accroissements radiaux moyens des arbres selon chaque variable liée à l'approvisionnement en eau : indice de réserve utile, classe de drainage, profondeur de sol. La réserve en eau utile dans le sol varie avec les caractéristiques édaphiques (texture, charge en cailloux...), mais également avec les possibilités d'apports latéraux d'eau par ruissellement. Ainsi, les sols les plus secs sont les sols les moins profonds ou caractérisés par un drainage latéral d'eau défavorable lié dans la majorité des cas, à une topographie du type haut de pente ou rebord de plateau. Ce résultat correspond bien aux observations de Décourt et Le Tacon (1974) pour le pin noir d'Autriche sur les plateaux calcaires du Nord-Est de la France. 
L'approvisionnement local en eau apparaît être également un facteur prédisposant aux effets des sécheresses. En effet, la divergence des courbes selon l'alimentation en eau du sol intervient nettement à la suite des années ou séquences d'années à déficits pluviométriques plus ou moins longs et marqués (fig 4). Ce résultat confirme les observations faites à la suite de l'étude entre l'aspect actuel des houppiers et la croissance radiale (Lebourgeois et Becker, 1996). La baisse de croissance observée dans les peuplements présentant actuellement des pertes foliaires est en relation directe avec une succession d'années particulièrement sèches, dont les effets ont été localement amplifiés par des conditions d'alimentation hydrique défavorables. Ces résultats, qui rejoignent les observations faites pour le sapin dans les Vosges (Becker et Lévy, 1988 ; Becker, 1991 ; Becker et al, 1995) et dans le Jura (Bert, 1993), et pour le chêne pédonculé dans la plaine d'Alsace (Luszczak-Clussmann, 1995), mettent une fois de plus l'accent sur l'importance du déterminisme climatique de nombreux phénomènes préoccupants de dépérissement observés actuellement, et sur l'importance des conditions stationnelles dans la sensibilisation des écosystèmes forestiers aux aléas climatiques.

L'analyse conjointe de la qualité de la nutrition et des conditions locales d'alimentation en eau (fig 5) montre que l'amélioration des niveaux d'accroissement est étroitement liée à la qualité de la nutrition, et que son effet est d'autant plus efficace que l'approvisionnement en eau est optimal. Ceci n'est pas surprenant car l'eau et les éléments minéraux sont indispensables simultanément pour de nombreux métabolismes (Sand et Mulligan, 1990). Cependant, il apparaît que les conditions climatiques peuvent moduler la réponse des arbres à la nutrition minérale. En effet, depuis la succession des années sèches de la décennie 1980, la croissance des peuplements bien alimentés au niveau minéral est fortement ralentie (figs 2, 3 et 5). Un tel phénomène a été déjà observé pour d'autres espèces telles que Picea abies (Spiecker, 1987), Abies alba (Becker, 1992), Pinus radiata (Turner, 1982 ; Linder et al, 1987), Pinus pinaster (Lemoine, 1993). Ces auteurs ont montré que la croissance des peuplements réagit positivement à la bonne disponibilité minérale pendant les périodes favorables du point de vue climatique, mais diminue fortement pendant une période de déficit hydrique. L'explication de ces comportements n'est pas évidente, et dépasse en partie le cadre de l'étude présentée ici. Cependant, les résultats de différents travaux sur Pinus radiata apportent certains éléments de réponse. En période climatique favorable, les arbres bien alimentés sur le plan minéral augmentent fortement leur surface foliaire (Linder et al, 1987), ainsi que leur « efficience » de croissance (rapport de la production en volume sur la masse foliaire) grâce à des taux de photosynthèse par unité de surface supérieurs (Linder et Rook, 1984). En revanche, en cas de sécheresse, ces arbres réagissent par une perte foliaire plus importante que les autres peuplements (Cromer et al, 1984 ; Linder et al, 1987), ce qui se traduit par une chute de la photosynthèse nette (Attiwil et Cromer, 1982) et, à terme, par une réduction de croissance. Un tel scénario pourrait s'appliquer dans notre cas. D'ailleurs, une étude écophysiologique menée parallèlement à ce travail a montré qu'une sécheresse édaphique estivale entraîne, chez des jeunes plants de pin laricio de Corse bien alimentés au plan minéral, une réduction notable des croissances aérienne et racinaire, en relation avec une diminution nette de l'assimilation carbonée. L'effet de la sécheresse s'exprime d'ailleurs l'année suivante par une réduction de la nouvelle pousse aérienne (Lebourgeois, 1995).

Il apparaît également que la moindre concurrence entre arbres peut minimiser l'effet de conditions hydriques locales a priori défavorables, particulièrement lors d'épisodes secs (fig 5). Un tel comportement a déjà été observé par Becker (1992) et 
Lebourgeois et al (1993) au cours d'expériences de fertilisation sur des sapinières adultes dans les Vosges. Ces auteurs ont montré que l'état de concurrence d'un arbre module clairement sa réponse aux apports minéraux et aux conditions environnementales de croissance. De la même façon, suite à une étude sur l'écologie du chêne sessile et pédonculé dans le Centre et le Nord-Est de la France, Lévy et al (1992) recommandent de maintenir une faible densité dans les peuplements localisés sur des stations particulièrement limitées au niveau hydrique. La réduction de la concurrence dans un peuplement (suite à une éclaircie par exemple) modifie favorablement de nombreux paramètres comme l'éclairement (dont l'un des effets directs est d'augmenter la photosynthèse), et la disponibilité en eau (ce qui se traduit par une diminution de l'intensité et de la durée du stress hydrique) (Aussenac et Granier, 1988 ; Bréda et al, 1995).

Les facteurs hydriques locaux, la nutrition minérale et le climat jouent donc un rôle très important dans l'expression de la croissance radiale. Si on peut remédier partiellement aux problèmes de carences minérales par des apports du ou des éléments limitants, on ne peut généralement pas modifier les conditions locales d'approvisionnement en eau. Ainsi, dans le futur, il serait raisonnable, pour ne pas sensibiliser les peuplements aux aléas climatiques, et éviter l'apparition des symptômes de dépérissement à plus ou moins long terme, de limiter les reboisements aux stations dont l'alimentation en eau n'est pas un facteur limitant essentiel, c'est-à-dire exclure les stations à topographie défavorable, les sols superficiels $(<40 \mathrm{~cm})$ ou caractérisés par un indice de réserve utile faible (inférieur à 40 par exemple). Le problème de la gestion des peuplements est plus délicat. On ne peut pas recommander un type de gestion particulier. Il s'agirait plutôt de moduler les régimes d'éclaircies selon les types de stations de façon à gommer quelque peu les contrastes naturels entre sites, et à minimi- ser la compétition pour l'eau lors de périodes à déficits pluviométriques importants.

Dans le cadre des effets des changements globaux sur les écosystèmes forestiers, et plus particulièrement de la variation probable en durée et en intensité des crises climatiques (hypothèse sérieusement envisagée en relation avec le réchauffement planétaire), il est d'ailleurs possible que la question de l'approvisionnement en eau au niveau de la placette joue dans les années à venir un rôle encore plus déterminant dans la production, voire dans la survie des peuplements.

\section{REMERCIEMENTS}

Les auteurs remercient le CRPF des Pays-de-laLoire (F Barbotin et B Guimbretière), l'équipe du Cemagref de Nogent-sur-Vernisson (R Chevalier, Y Dumas, JM Gilbert et B Vallée), ainsi que les techniciens de l'unité d'écophysiologie (F Gérémia, R Schipfer, D Himbert) pour l'aide apportée lors de la récolte des données sur le terrain.

\section{RÉFÉRENCES}

Agrawal DC (1988) Effect of phosphorus nutrition on some primary and secondary plant metabolites in Pintus caribae Mor seedlings. Indian J For 11 , 272-275

Attiwill P. Cromer RN (1982) Photosynthesis and transpiration of Pintes radiata (D Don) under plantation conditions in southern Australia. I. Response 10 irrigation with waste water. Aus J Plant Phssiol 9.749-760

Aussenac G, Granier D (1988) Effects of thinning on water stress and growth in Douglas-fir. Can J For $\operatorname{Res} 18.1 .100-105$

Badeau V, Dupoucy JL, Becker M, Picard JF (1995) Long term growth trends of Fagus svlvatica $\mathrm{L}$ in Northeastern France. A comparison between High and Low density stands. Acta Recol 16, 571-583

Becker M (1989) The role of climate on present and past vitality of silver fir forests in the Vosges mountains of northeatstern France. Can J For Res 19. $1110-1117$

Becker M (1991) Incidence des conditions climatiques, édaphiques ct sylvicole sur la croissance et la santé des forêts. In : Les recherches en France sur le 
dépérissement des forêts, Programme Deforpa. Deuxième rapport. Engref, 25-41

Becker M (1992) Radial growth of mature silver firs (Abies alba Mill) fertilized in 1969. Interaction of climate and competition. International Dendrochronological Symposium Tree rings and Environment, Lund, Sweden, 17-21

Becker M, Bert GD, Landmann G, Lévy G, Rameau JC, Ulrich E (1995) Growth and decline symptoms of Silver Fir and Norway Spruce in Northeastern France : Relation to climate, nutrition and silviculture. Pages 120-142. In: Forest Decline and Atmospheric Deposition Effects in the French Mountains (G Landmann and M Bonneau, eds), Springer, Berlin, $461 \mathrm{p}$

Becker M, Lévy G (1988) À propos du dépérissement des forêts : climat, sylviculture et vitalité de la sapinière vosgienne, Rev For Fr 40, 345-358

Bert GD (1993) Impact of ecological factors, climatic stresses, and pollution on growth and health of silver fir (Abies alba Mill) in the Jura mountains: an ecological and dendrochronological study. Acta Ecol 14, 229-246

Bonneau M (1963) Premiers résultats d'une expérience de fertilisation sur pin laricio de Corse en forêt de Moulière (86). Ann Ec Nat Eaux Forêts Rech Exp For 20, 314-341

Bonneau M (1970) Résultats après 9 ans de l'essai de fertilisation de Moulière (Vienne). Ann Sci For 27, 111-125

Bonneau M (1980) Cours de pédologie forestière. $2^{\mathrm{e}}$ partie, chapitre $\mathrm{V}$ : appréciation de la fertilité du sol ; éléments autres que l'azote. Nancy : Engref, $26 \mathrm{p}$

Bonneau M (1988) Le diagnostic foliaire. Rev For Fr $40, n^{\circ}$ spécial, $19-28$

Breda N, Granier A, Aussenac G (1995) Effects of thinning on soil water balance and trees water relations, transpiration and growth in an oak forest (Quercus petraea). Tree Physiol 15, 295-306

Briffa KR (1992) Increasing productivity of 'natural growth' conifers in Europe over the last century. International Dendrochronological Symposium 'Tree rings and Environment', Lund University Department of Quaternary Geology 34, 64-71

Cromer RN, Tompkins D, Barr NJ, Williams ER, Stewart HTL (1984) Litter fall in a Pinus radiata forest: the effect of irrigation and fertilizer treatments. J Appl Ecol 21, 313-326

Dambrine E, Le Goaster S, Ranger J (1991) Croissance et nutrition minérale d'un peuplement d'épicéa sur sol pauvre. II. Prélèvement racinaire et translocation d'éléments minéraux au cours de la croissance. Acta Ecol 12, 791-808

Debazac EF (1964) Le pin laricio de Corse dans son aire naturelle. Rev For Fr 16, 188-215

Decourt N (1973) Production primaire, production utile : méthodes d'évaluation, indices de productivité. Ann Sci For 30, 219-238
Decourt N, Le Tacon F (1974) Comportement du pin noir d'Autriche (Pinus nigra Arn ssp nigricans) sur les plateaux calcaires de l'est de la France. $R e v$ For Fr 26, 211-218

Duhamel D (1984) Statut écologique du chêne pédonculé et du chêne sessile dans le quart nord-ouest de la France. Performances relatives et gestion sylvicole. Mémoire, Engref-Inra, Nancy, $58 \mathrm{p}$

Dupouey JL, Denis JB, Becker M (1992) A new method of standardization for examining long term trends in tree-ring chronologies. International Dendrochronological Symposium 'Tree rings and Environment', Lund University Department of Quaternary Geology 34, 85-88

Dure CE, Charon D (1994) Pin laricio. Forêt-Entreprise $96,70-72$

Fritts HC (1976) Tree-ring and climate. Academic Press, New York, $576 \mathrm{p}$

Guitton JL, Bonneau M, Adrian M (1988) Résultats de fertilisation minérale en région méditerranéenne. Rev For Fr 40, 315-320

Helmisaari HS (1992) Nutrient retranslocation within foliage of Pinus sylvestris. Tree Physiol 10, 45-58

Lebourgeois F (1995) Étude dendroécologique et écophysiologique du pin laricio de Corse (Pinus nigra Arnold ssp laricio Poiret var corsicana) en région Pays-de-la-Loire. Thèse, université de Paris-SudOrsay, $209 \mathrm{p}+$ annexes

Lebourgeois F, Becker M (1996) Dendroécologie du pin laricio de Corse dans l'Ouest de la France. Évolution du potentiel de croissance au cours des dernières décennies. Ann Sci For, 53, 931-946.

Lebourgeois F, Becker M, Bonneau M (1993) Influence d'une fertilisation minérale sur la croissance radiale de sapinières dépérissantes dans les Vosges. Rev For Fr 55, 639-650

Le Goaster S, Dambrine E, Ranger J (1991) Croissance et nutrition minérale d'un peuplement d'épicéa sur sol pauvre. I. Évolution de la biomasse et dynamique d'incorporation d'éléments minéraux. Acta CEcol 12.771-789

Le Goff N, Lévy G (1984) Productivité du frêne (Fraxinus excelsior $\mathrm{L}$ ) en région Nord-Picardie. B. Étude des relations entre la productivité et les conditions du milieu. Ann Sci For 4 1, 135-170

Lemoine P (1993) Étude dendroécologique du pin maritime (Pinus pinaster Ait) sur le dispositif de fertilisation de Mimizan (Landes). Mémoire de DEA, université de Nancy I, 22 p + annexes

Lévy G (1988) Appréciation de la fertilité du sol. Rev For Fr 40, n ${ }^{\circ}$ spécial, 1 1-17

Lévy G, Becker M, Duhamel D (1992) A comparison of the ecology of pedunculate and sessile oaks: radial growth in the centre and northwest of France. For Ecol Manag 55, 51-63.

Linder S, Benson ML, Myers BJ, Raison RJ (1987) Canopy dynamics and growth of Pinus radiata. $\mathbf{I}$. Effects of irrigation and fertilization during a drought. Can J For Res 17, 1157-1165 
Linder S, Rook DA (1984) Effects of mineral nutrition on carbon dioxide exchange and partitioning of carbon in trees. In: 'Nutrition of Plantation Forests' (GD Bowen, EKS Nambiar, eds), Academic Press, London, 21 1-236

Luszczak-Clussmann G (1995) Étude dendroécologique du chêne pédonculé (Quercus robur $\mathrm{L}$ ) et du frêne commun (Fraxinus excelsior L) en forêt d'Illwald (Bas-Rhin). Mémoire de DESS, université de Strasbourg, $47 \mathrm{p}+$ annexes

McNeil RC, Ballard R, Duzan HW (1984) Prediction of mid-term from short-term fertilizer responses for southern pine plantations. Forest Sci 30, 264-269

Mesanza JM, Casado H, Castillo FJ (1993) Nutrient concentrations in Pinus radiata needles in the Basque Country (Spain): a preliminary classification of parameters and sites. Ann Sci For 50, 23-36.

Miller HG (1986) Carbon $\times$ nutrient interactions - the limitations to productivity. Tree Physiol 2, 373385

Miller HG, Miller JD (1976) Effect of nitrogen supply on net primary production in Corsican pine. $J$ Appl Ecol 13, 249-256

Miller HG, Miller JD, Cooper JM (1980) Biomass and nutrient accumulation at different growth rates in thinned plantations of Corsican pine. Forestry 53 , $1,23-39$

Miller HG, Miller JD, Cooper JM (1981) Optimum foliar nitrogen concentration in pine and its change with stand age. Can J For Res 1 1, 3, 563-572

Moffat AJ, Matthews RW, Hall JE (1991) The effects of sewage sludge on growth and foliar and soil chemistry in pole-stage Corsican pine at Ringwood Forest, Dorest, UK. Can J For Res 21, 902-909

Nambiar EKS, Fife DN (1991) Nutrient retranslocation in temperate conifers. Tree Physiol 9, 185-207

Nys C, Bonischot R, Gelhaye D (1985) Réponse d'un peuplement de pin laricio de Corse (Pinus nigra espèce Laricio) à la fertilisation en Sologne. Rev For Fr 37 (6), 482-486

Parde J, Bouchon J (1988) Dendrométrie. $2^{e}$ édition. Engref, Nancy, $328 \mathrm{p}$

Prevot P, Ollagnier M (1956) Méthode d'utilisation du diagnostic foliaire. In : Analyse des plantes et problème des fumures minérales. Édition IRHO, 117-192

Proe MF. Dutch J, Miller HG, Sutherland J (1992) Long-term partitioning of biomass and nitrogen following application of nitrogen fertilizer to Corsican pine. Can J For Res 22, 82-87

Raison RJ, Khanna PK, Connel MJ, Falkiner RA (1990) Effects of water availability and fertilization on nitrogen cycle in a stand of Pinus radiata. For Ecol Manag 30, 31-43

Ranger J (1978) Recherches sur les biomasses comparées de deux plantations de pin laricio de Corse avec ou sans fertilisation. Ann Sci For 35, 93-115
Ranger J (1981) Étude de la minéralomasse et du cycle biologique dans deux peuplements de Pin laricio de Corse dont l'un a été fertilisé à la plantation. Ann Sci For 38, 127-158

Raupach M, Boardman R, Clarke ARP (1969) Growth rate of Pinus radiata (D Don) in relation to foliar levels of nitrogen and phosphorus for plantations in south-east of South Australia. In: Soil Publ N ${ }^{\circ} 26$, CSIRO, Australia

Roman-Amat B (1984) Contribution à l'exploration et à la valorisation de la variabilité intraspécifique et individuelle du pin laricio de Corse, Pinus nigra Arnold ssp laricio var corsicana Loud. Thèse de l'université d'Orsay, n ${ }^{\circ}$ 679, 144 p

Roman-Amat B, Arbez M (1985) Pin laricio de Corse et de Calabre. Quelles provenances choisir? Le point sur les expériences comparatives de l'Inra. Rev For Fr 37, 377-388

Sand R, Mulligan DR (1990) Water and nutrient dynamics and tree growth. For Ecol Manag 30, 91-111

Sheriff DW, Nambiar KS, Fife DN (1986) Relationships between nutrient status, carbon assimilation and water use efficiency in Pinus radiata (D Don) needles. Tree Physiol 2, 73-88

Smith CJ, Wellwood RW, Elliot J (1977) Effects of nitrogen fertilizer and current climate on wood properties of Corsican pine (Pinus nigra var maritima (Ait) Melv). Forestry 50, 117-138

Spiecker H (1987) Düngung, Niederschlag und der jährliche Volumenzuwachs einiger Fichtenbestände. Südwestdeutschlands. Ergebnisse einer neuen Methode der Zuwachsermittlung. Allg Forst - $u \mathrm{~J}$. $\mathrm{Ztg}, 158 \mathrm{Jg}, 4,70-76$

Tessier $L$ ( 1989) Spatio-temporal analysis of climate-tree ring relationships. New Phytol 111,517 529

Turner J (1982) Long-term superphosphate trial in regeneration of Pinus radiata at Belanglo State Forest, NSW Aust For Res, 12, 1-9

Ung CH (1989) Forme des tiges de l'érable à sucre et du hêtre à grandes feuilles dans une jeune futaie selon leur position sociale et leur âge. Ann Sci For 46, 261-271

Valentine DW, Allen HL (1990) Foliar responses to fertilization identify nutrient limitation in loblolly pine. Can J For Res 20, 144-151

Visser H (1995) Note on the relation between ring widths and basal area increments. For Science 41, 297-304

Yaric J, Van Cleve K, Schlentner R (1990) Interaction between moisture, nutrients and growth of white spruce in interior Alaska. For Ecol Manag 30, 73-89.

Zarnovican R, Trencia J (1987) Croissance de l'érable à sucre et du hêtre à grandes feuilles selon la position sociale dans le peuplement. Ann Sci For 44, $211-226$ 